\title{
The Regression Analysis through Matlab of the Electrical Parameters in a Hydropower Plant
}

\author{
Virtyt Lesha \\ Polytechnic University of Tirana, Albania
}

\section{Abstract}

This paper presents the nonlinear regression analysis between the transformer's measurable parameters (temperature of oil and winding) and the electrical parameters (hydropower plant's aggregate). There is not a specific mathematical function that connects the winding and oil temperature with the generator voltage, generator current, active power, reactive power, etc. Therefore to find the connection between them, statistical methods are needed. So, the work in this article consists in putting as a position variable of the electrical parameters of an aggregate in a hydropower plant against the parameters such as transformer's winding and oil temperature. Through Matlab, these data are analyzed through the sixth order nonlinear polynomial regression giving the determination coefficients. he reason for the use of the nonlinear regression of this order is related to the fact that the use of nonlinear regression of higher orders does not represent more accurate apparent than nonlinear regression of higher orders. On the other side the regressions with lower orders has not been used because their accuracy is significantly lower than the order of regression in question.

Keywords: technology, Matlab, nonlinear regression, winding temperature, oil temperature

JEL classification: C20, L9

\section{Introduction}

A power transformer is a static device consisting of one, two or more windings, which can be either with a magnetic core to induce common coupling between the circuits. The transformers are widely used in electrical power systems for transmitting electromagnetic induction between the circuits with the same frequency and usually this happens with variable values of the voltage and electric current. (Winders, 2002)

The power transformers are needed to connect parts of the power system operating at different voltage levels (Saadat, 1999). Besides changing the voltage level, transformers are used, as well as to control the voltage control and are equipped with cock in one or more winding to allow the changing of the order.

The generation and transmission transformers have ranges of severe of dozens of megavolt-ampere to more than 1000 MVA and are usually cooled with oil. Transformer core is located within an oil tank which acts as a cooling but also for as an isolator for the windings of the transformer (Wang, 2009). Heat, due to the loss of core as well as the ohm loss itself in the transformer windings, is eliminated from the oil through external radiators. Turnover in the transformer oil is natural or forced. Air circulation outside the transformer usually is stimulated through the ventilators. Because of transportation problems, the large power transformers are usually constructed as three single-phase 
separate transformers. Small transformers are usually of a three-phase integrated design (Heathcote, 1998).

The goal of the paper consists in defining the trend of the data obtained from the VauDejes hydropower plant. These include the temperature of oil and winding of the transformer as well as the electrical parameters mentioned below. The structure of the paper begins with methodology used to make the nonlinear regression analysis. Then the results of this analysis are presented. Finally after showing and interpreting these results, the section of conclusion, recommendation and future work is treated.In this paper many different sources were used such as Machowski et al. (2008), Saadat (1999), Wang (2009), Sivanadam (2007) and more.

\section{Methodology and data}

In this study we have taken samples from the interval 14.07.2014-29.08.2014. So, for 47 days, every hour of the day for 20 consecutive hours of electrical parameters of the aggregate we have made the absorption of the data from the computer control room of the VauDejes hydropower plant. Then, for the data of each day, the mathematical average of these parameters is calculated. These parameters are:

- The generator voltage

- The generator current

- The active power of the generator

- The reactive of power generator

- The excitation voltage

- The excitation current

These electrical parameters of the aggregate are set in the depending of the temperature of the transformer oil and winding. We know that the higher the values of these electrical parameters in the aggregate are, the greater is the temperature of the oil and winding in the transformer, but, a functional link does not exist. Consequently, the only way to find the dependence from each other is the statistical study. In this study we used nonlinear polynomial regression of the sixth order to find this dependence.

\section{The results of the simulations}

In this section we will look at the functional connections of the above mentioned electrical parameters of the generator of an aggregate versus the transformer's temperature of winding and oil in that aggregate (Sivanadam, 2007). 
Figure 1

(a) The graph that expresses the dependency between the temperature of the winding and the voltage of the generator and the nonlinear regression polynomial curve of sixth order

(b) The graph that expresses the dependency between the temperature of the oil and the voltage of the generator and the nonlinear regression polynomial curve of sixth order

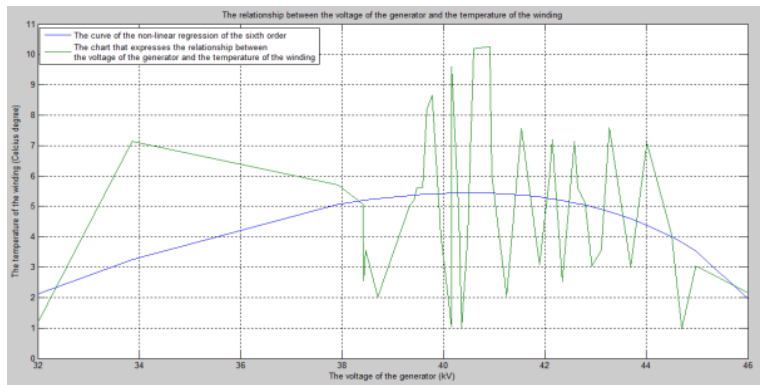

(a)

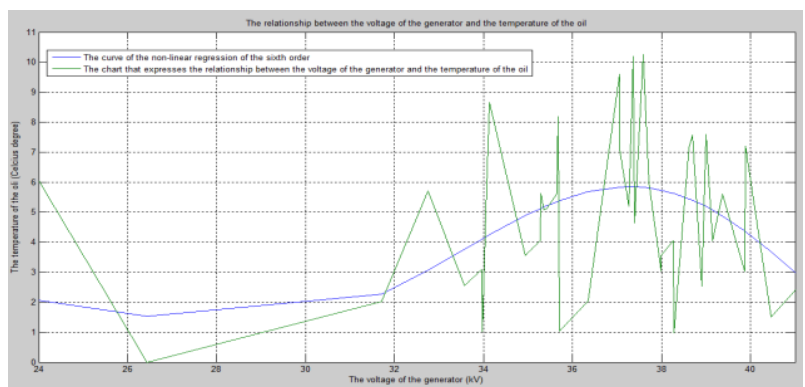

(b)

Source: Matlab programming results

In the figure $1(a)$, the temperature of the winding depends on the generator voltage to the extent of $\mathbf{2 . 2 2 9 1 \%}$. The rest of dependence remains to be studied.

In the figure $1(\mathrm{~b})$, the temperature of the oil depends on the generator voltage to the extent of $\mathbf{2 . 2 9 8 8 \%}$. The rest of dependence remains to be studied

Figure 2

(a)The graph that expresses the dependency between the temperature of the winding and the current of the generator and the nonlinear regression polynomial curve of sixth order

(b) The graph that expresses the dependency between the temperature of the oil and the current of the generator and the nonlinear regression polynomial curve of sixth order

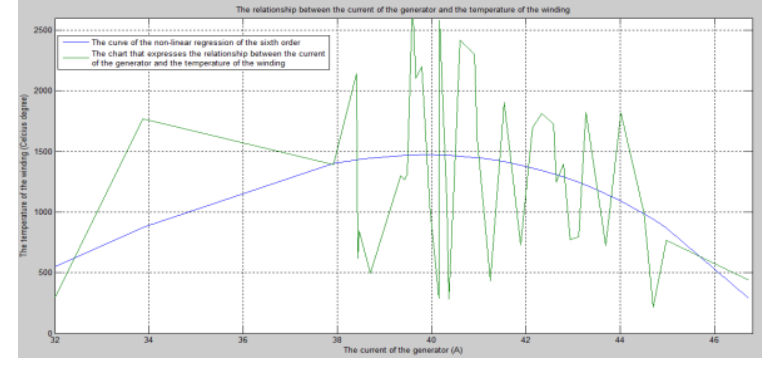

(a)

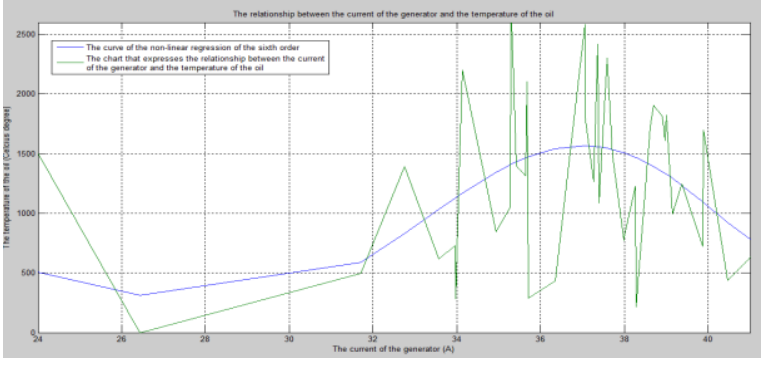

(b)

Source: Matlab programming results

In the figure 2 (a), the temperature of the winding depends on the generator current to the extent of $\mathbf{5 . 4 7 8 1 \%}$. The rest of dependence remains to be studied.

In the figure 2 (b), the temperature of the oil depends on the generator current to the extent of $\mathbf{6 . 0 7 5 8 8 \%}$. The rest of dependence remains to be studied. 
Figure 3

(a) The graph that expresses the dependency between the temperature of the winding and the active power of the generator and the nonlinear regression polynomial curve of sixth order

(b) The graph that expresses the dependency between the temperature of the oil and the active power of the generator and the nonlinear regression polynomial curve of sixth order

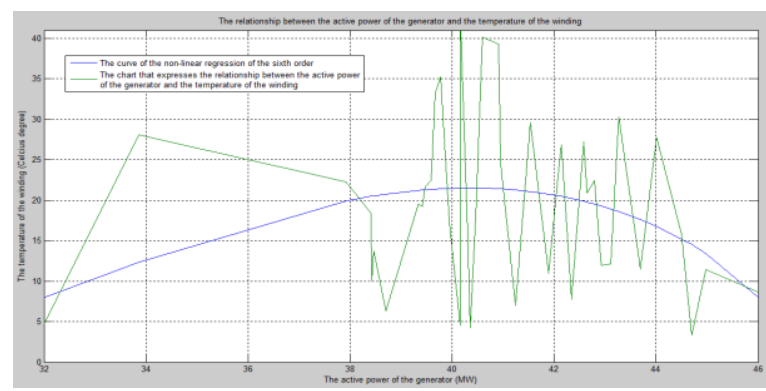

(a)

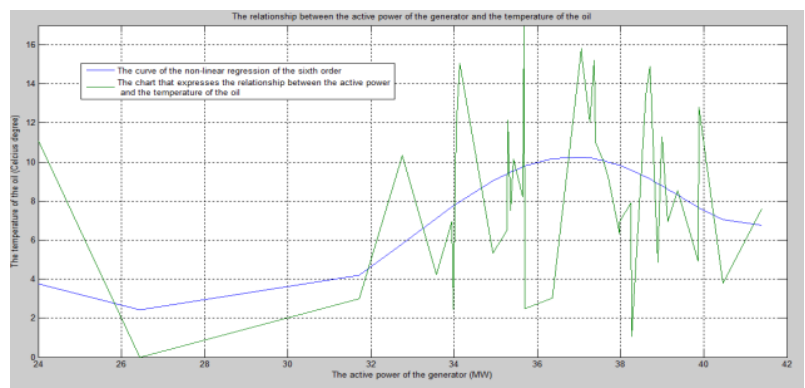

(b)

Source: Matlab programming results

In the figure $3(a)$, the temperature of the winding depends on the generator active power to the extent of $\mathbf{9 . 1 3 5 3 \%}$. The rest of dependence remains to be studied.

In the figure 3 (b), the temperature of the oil depends on the generator active power to the extent of $\mathbf{3 . 9 7 4 \%}$. The rest of dependence remains to be studied.

Figure 4

(a) The graph that expresses the dependency between the temperature of the winding and the reactive power of the generator and the nonlinear regression polynomial curve of sixth order

(b)The graph that expresses the dependency between the temperature of the oil and the reactive power of the generator and the nonlinear regression polynomial curve of sixth order

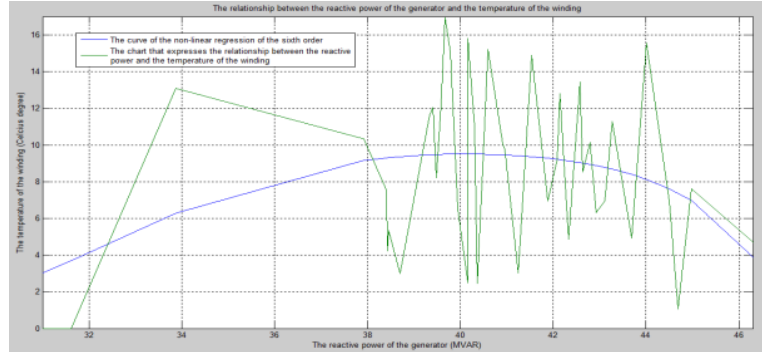

(a)

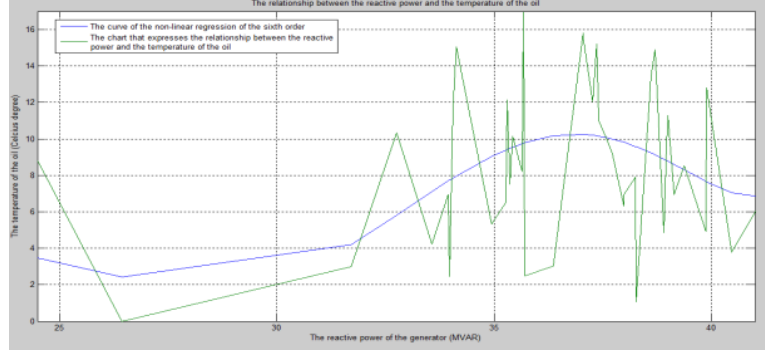

(b)

Source: Matlab programming results

In the figure $4(\mathrm{a})$, the temperature of the winding depends on the generator reactive power to the extent of $\mathbf{3 . 8 4 0 5 \%}$. The rest of dependence remains to be studied.

In the figure 4 (b), the temperature of the oil depends on the generator reactive power to the extent of $3.974 \%$. The rest of dependence remains to be studied. 
Figure5

(a) The graph that expresses the dependency between the temperature of the winding and the excitation voltage of the generator and the nonlinear regression polynomial curve of sixth order

(b) The graph that expresses the dependency between the temperature of the oil and the excitation voltage of the generator and the nonlinear regression polynomial curve of sixth order.
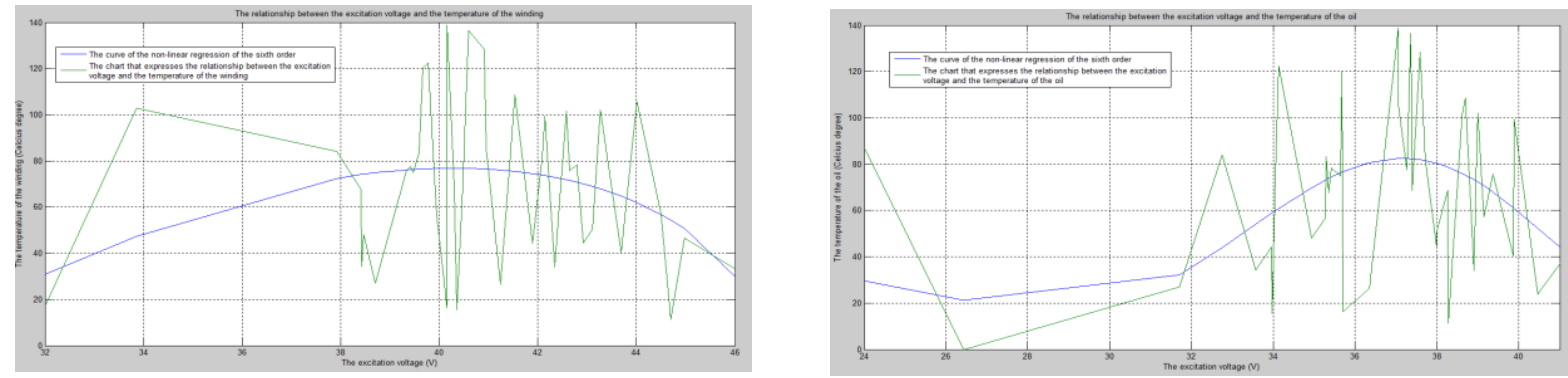

(a) (b)

Source: Matlab programming results

In the figure $5(\mathrm{a})$, the temperature of the winding depends on the generator excitation voltage to the extent of $\mathbf{3 . 0 9 9 1 8 \%}$. The rest of dependence remains to be studied.

In the figure $5(\mathrm{~b})$, the temperature of the oil depends on the generator excitation voltage to the extent of $\mathbf{3 . 2 0 2 1 3}$. The rest of dependence remains to be studied.

Figure 6

(a) The graph that expresses the dependency between the temperature of the winding and the excitation current of the generator and the nonlinear regression polynomial curve of sixth order

(b) The graph that expresses the dependency between the temperature of the oil and the excitation current of the generator and the nonlinear regression polynomial curve of sixth order.

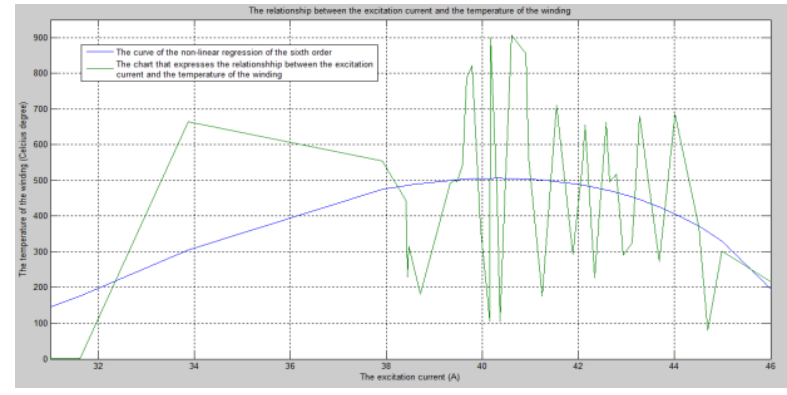

(a)

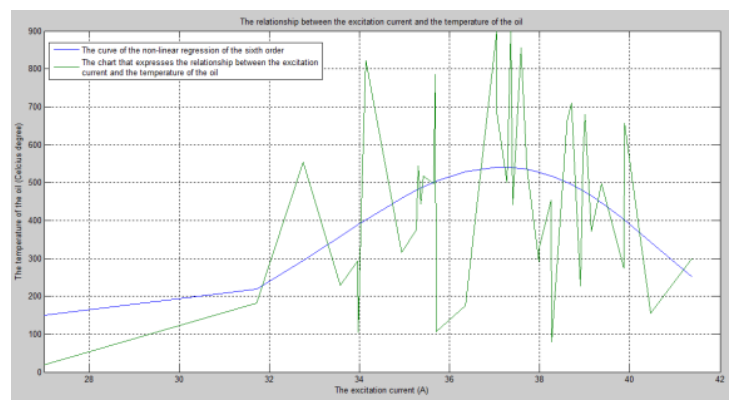

(b)

Source: Matlab programming results 
In the figure 6(a), the temperature of the winding depends on the generator excitation voltage to the extent of $\mathbf{2 . 0 3 0 5 3 2 \%}$. The rest of dependence remains to be studied.

In the figure $6(\mathrm{~b})$, the temperature of the oil depends on the generator excitation voltage to the extent of $\mathbf{2 . 1 0 3 2 1 9}$. The rest of dependence remains to be studied.

Table 1

The over view of the Dependencies of the Parameters to the Coefficients of Determination

\begin{tabular}{cc}
\hline Dependencies & $\begin{array}{c}\text { The coefficient of the } \\
\text { determination (R) }\end{array}$ \\
\hline Winding temperature - Generator voltage & 0.022291 \\
\hline Oil temperature - Generator voltage & 0.022988 \\
\hline Winding temperature - Generator current & 0.054781 \\
\hline Oil temperature - Generator current & 0.0607588 \\
\hline Winding temperature - Generator active power & 0.0911353 \\
\hline Oil temperature - Generator active power & 0.03974 \\
\hline Winding temperature - Generator reactive power & 0.038405 \\
\hline Oil temperature - Generator reactive power & 0.03974 \\
\hline Winding temperature - Generator excitation voltage & 0.0309918 \\
\hline Oil temperature - Generator excitation voltage & 0.0320213 \\
\hline Winding temperature - Generator excitation current & 0.02030532 \\
\hline Oil temperature - Generator excitation current & 0.02103219 \\
\hline
\end{tabular}

Source: Results generated from the data of the above graphs

\section{Conclusions}

In this article we gave the results of the simulation via Matlab (Katsikis, 2012) of the dependency of the generator's electric parameters of an aggregate in a hydropower plant versus the temperature of winding and oil of a power transformer of this aggregate. According to the theory of transformers we are aware that as greater the energy held by the aggregate, the greater the temperature of the winding and oil of the power transformer is. However, we can say that a precise mathematical relation between temperature of the oil and winding of the power transformer and the electrical parameters of the generator does not exist.

Therefore, we have used statistical methods of the data processing which provide a continuity trend of these data and this trend is reflected in an equation of line which may be linear or not, and a coefficient of determination. This coefficient is an indicator that shows the order of dependency of data from each other.

The first results of this paper have consisted in presenting the regressive dependency of the generator voltage versus temperature of the winding and oil of this aggregate. Then, we have performed simulations in Matlab (Katsikis, 2012) for the regressive reflection of other parameters of the aggregate. The parameters considered, in this article are, the generator voltage, the generator current, the generator active power and the generator reactive power. It should be emphasized that these parameters have been at the position of the variable, which lie in the function of the temperature of the winding and oil of the transformer. 
From the table of the coefficients of determination we observe that the order of dependency of the electrical parameters of the transformer temperature is relatively low, which means it does not exceed 0.0911353. This result is evident because the sampling time was during the summer season and the aggregate did not operate with full force due to lack of rainfall. This means that convey energy from the generator to the transformer has been relatively small and this is reflected in the winding and oil temperature of the power transformer.

The limitations of this paper are related to the fact that we have used the nonlinear regression method of the sixth order to give the tendency of these data which may lead to a prediction of this dependency. The reason for the use of the nonlinear regression of this order is related to the fact that the use of nonlinear regression of higher orders does not represent more accurate apparent than nonlinear regression of higher orders. On the other side the regressions with lower orders has not been used because their accuracy is significantly lower than the order of regression in question.

Finally I propose as future work the use of nonlinear regression of higher degrees, such as affecting the results so that to show more correct approximations to these kind of parameters.

\section{References}

1. Heathcote, M., (1998), "The J \& P transformer book", Newnes, pp. $346-353$.

2. Katsikis, V. (2012), "Matlab - a fundamental tool for scientific computing and engineering applications", InTech, pp. 17-31.

3. Katsikis, V. (2012), "Matlab - a fundamental tool for scientific computing and engineering applications", InTech, pp.153-170.

4. Machowski, J., Bialek, J., Bumby, J., (2008), "Power system dynamics: stability and control", Wiley, pp. 103-106.

5. Saadat, H. (1999), "Power system analysis", McGraw-Hill, pp. 126-142 .

6. Sivanadam, S., Sumathi, S., Deepa, S., (2007), "Intruction to fuzzy logic using Matlab", Springer, pp. 65-72.

7. Wang, L. (2009), "Model predicive control system design and implementation using Matlab", Springer, pp. 336-338.

8. Winders, J. (2002), "Power transformers: principles and applications", Marcel Dekker, pp. 6879.

\section{About the author}

Virtyt Lesha is finishing the Master of Science studies in Electronics Engineering at Polytechnic University of Tirana. One of his interests includes the designing of automatic control systems through Matlab/Simulink. During the studies he has done an internship at the Hydroelectric Power Station of Vau-Dejes in Shkodra, Albania. In this power station he has analyzed the control systems of the 5 turbines in this central. His master thesis will consist in the modeling through Matlab/Simulink of the hydro-turbine, the generator and the power transformer of an aggregate of the abovementioned hydroelectric central. He has 7 publications in international conferences. Virtyt Lesha can be contacted at virtytl@hotmail.com 